\title{
Informing primary care physicians of patients' involvement in clinical trials carried out at a specialist care level
}

This article was published in the following Dove Press journal:

Open Access Journal of Clinical Trials

27 July 2017

Number of times this article has been viewed

\author{
Joan Antoni \\ Schoenenberger-Arnaiz ${ }^{1,2}$ \\ Montserrat \\ Solanilla-Puertolas ${ }^{2}$ \\ Maria Acer-Puig ${ }^{3}$ \\ Javier Gomez-Arbones 3,4 \\ 'Pharmacy Department, Arnau \\ de Vilanova University Hospital, \\ ${ }^{2}$ Institutional Review Board, Arnau \\ de Vilanova University Hospital, \\ ${ }^{3}$ Department of Medicine, Faculty \\ of Medicine, University of Lleida, \\ ${ }^{4}$ IRB Lleida, University of Lleida, \\ Arnau de Vilanova University Hospital, \\ Lleida, Spain
}

Background: Patients enrolled in clinical trials continue to have frequent contacts with primary care physicians because of comorbidities or toxicities. The aim of the present study was to analyze the information provided at different levels, when participants are included in clinical trials organized at a specialized care level. The purpose was to verify if informing the patient's primary care physician is contemplated in the inclusion process.

Methods: The authors conducted a cross-sectional study that included the clinical trials approved in the last 2 years by the hospital's Institutional Review Board. In addition, some of the participants in the included clinical trials were interviewed in order to check their knowledge of the type of research taking place.

Results: In total, 67 protocols and the accompanying informed consent documents were reviewed. Half of the reviewed protocols (48\%) did not provide participants with an identification card. Regarding the role of the primary care physician, $68.6 \%$ of clinical trials (46/67) had taken it into account in different ways. In only four trials, the method used to contact the primary care physician was documented. In total, 20 participants were interviewed. Only 3 (15\%) knew the title of the study in which they were participating, 14 (70\%) were aware of their illness and $6(30 \%)$ did not know how to answer any of these two questions. Almost all participants in the study knew the name of the physician who was the principal investigator in the trial.

Conclusion: Information given to health care practitioners, who are not directly involved in clinical trials conducted by specialized medical staff, is still scarce. In our clinical setting, patients participating in clinical trials have a low awareness of such studies.

Keywords: informed consent, clinical trials, family physician, wallet card

\section{Introduction}

Most medical patients are treated in the primary care settings, while most of the patients included in clinical trials are treated and monitored within the academic setting. ${ }^{1,2}$ In contrast, in our clinical setting the majority of cancer patients are treated in specialized clinics and oncologists are responsible for the enrollment of these patients in clinical trials. However, all patients enrolled in clinical trials, including cancer patients, continue to have frequent contacts with primary care physicians because of comorbidities, toxicities or needs of additional information. This is why primary care physicians have to be aware of the enrollment of their patients in clinical trials. Moreover, good clinical practice (GCP) guidelines recommend that, when conducting a trial, the patient's primary care physician should be informed about the subject's enrollment in the study. This would only happen after obtaining the subjects consent to release this information. Clinical trial enrollment is a key information for any health Schoenenberger-Arnaiz Pharmacy Department, Arnau de Vilanova University Hospital and IRB Lleida, Avda Alcalde Rovira Roure 80, Lleida 25198, Spain

Tel +34973705220

Fax +34 973234790

Email schoenenberger3573@gmail.com 
care professional, but especially for those who can prescribe drugs with a potential to interact with study medications or those who might care for the patient in emergency facilities.

Clinical investigators, and especially oncologists, need to communicate more effectively with primary care physicians if they are to expect their cooperation in referring potential subjects that could be considered for enrollment in a clinical trial. Furthermore, the primary care team is very well placed for monitoring how well patients are responding to cancer treatments. There needs to be a mechanism to help inform doctors about what clinical trials that are available to their patients and what are the selection criteria that are available for those trials in their catchment area. ${ }^{3,4}$ Means for obtaining information regarding questionable methods for treating cancer should be more easily accessible. ${ }^{4}$

In particular, clinical research requires a multidisciplinary team, and a primary care physician must be part of that team. Ensuring the successful development and conduct of clinical trials in practice-based research networks (PBRN), requires a highly collaborative approach between academic research experts and PBRN teams. ${ }^{5}$ Trial participation is very often limited by the primary care physician's lack of awareness of the available clinical trials for patients treated in their area of practice. According to a 2004 survey conducted by Taylor, the most common reason given by physicians when explaining nonparticipation in clinical trials was a lack of knowledge of their existence. ${ }^{6}$ Furthermore, primary care and specialized consultants who are not in partnership with research institutions might be even less aware of a patient's eligibility for inclusion in a clinical trial. ${ }^{7}$ Physicians are the main channel for patient entry into a clinical trial and, therefore, their knowledge and endorsement of the trials is essential in the enrollment of subjects. ${ }^{8}$

The primary resources, useful in locating information about clinical trials, are the clinical trial registries available to patients and health care professionals. ${ }^{7}$ There are a number of registries that set different goals depending on the intended user. The most comprehensive public registry to date is ClinicalTrials.gov, since February 2000, where all entities conducting trials with experimental treatments are required to submit information. The specific information requested for registration with ClinicalTrials.gov was established by the National Library of Medicine of the US Department of Health and Human Services as a result of Section 113 of the FDA Modernization Act of 1997. However, public awareness of clinical research coming from other sources is increasing ${ }^{8,9}$ and there is a need to overcome misconceptions regarding clinical trials. ${ }^{10,11}$
On another matter, recruitment methods are varied. The extended system for communicating clinical trial participation consists of a referral letter addressed to the clinicians asking them to recruit subjects from their own population of patients and to refer potential ones. Letters addressed to the treating clinician do not usually have to be approved by the Institutional Review Board (IRB); however, other recruitment methods and means (letters, flyers, posters, etc.) used to contact potential subjects must be approved by the IRB. It is also advisable to include an IRB approved study summary and/or brochure(s) with this referral letter.

The aim of the present study was to analyze the informed consent of a series of clinical trials. The main objective was to confirm that making the primary care physicians aware of the inclusion of one of their patients in a trial is in the informed consent.

In addition, the authors checked for the existence of a personal patient card which identified the participants in the clinical trial; they also ascertained whether this document contained useful information for practitioners who are not included in the research team. The present research was promoted by the "Comite Ėtic d'Investigació de l'Hospital Universitari Arnau de Vilanova" and approved by the same IRB. The participants interviewed provided written informed consent when included in the clinical trials that were analyzed.

\section{Methods}

The authors conducted a cross-sectional study that included the trial protocol (specifically the informed consent form) for clinical trials approved in the last 2 years by the hospital IRB. This documentation was reviewed and the following variables were calculated:

1. The proportion of clinical trials in which a personal identification card (ID) is provided to participants, and this is specified in the informed consent form. The ID must contain the title of the study, identification and contact details for the principal investigator (PI), and the most important safety considerations about the study.

2 . The proportion of trials in which informed consent provides the option to inform the general practitioner about the participation in the clinical trial.

3. Among trials that specify the option to inform the patient's general practitioner, the authors sought to identify who was in charge for this communication.

The authors also interviewed some of the participants in the included clinical trials to check their understanding of the research that was taking place and in which they were participating, as 
well as their feelings related to trial participation. The complete text of the survey is provided in Figure S1. Once included in a clinical trial, participants would be referred to the clinical trial pharmacy service so that the experimental medication could be dispensed. It was at this point, during the dispensing visits in pharmacy, that the participants would be interviewed.

\section{Results}

In total, 67 protocols and the accompanying informed consent documents were reviewed; the majority of studies were specialized in oncology $(33 / 67 ; 49.25 \%)$ but other fields, such as cardiology or endocrinology, were also involved. Most of the trials were Phase II or III (63/67; 94\%).

\section{Clinical trial protocols and information for the general practitioner}

As is displayed in Figure 1, nearly half of the reviewed protocols $(48 \%)$ did not provide participants with an ID card. Among those who did provide an ID card, a significant proportion $(37 \%)$ did not mention this card and its utility in the informed consent form.

Regarding the role of the primary care physician, $68.6 \%$ of clinical trials (46/67) had considered this in different ways and did refer to it in the informed consent. In contrast, in $31.3 \%(21 / 67)$ of the protocols there was no reference at all. Among trials that mentioned the role of the primary care physician, the methods used to communicate the inclusion of the patient in the clinical trial are shown in Figure 2. In most cases, the informed consent stated that the patients were agreeing to allow medical staff involved in the study to contact their primary care physician. However, in only four trials the specific method used for that purpose was indicated, usually through an informative letter.

\section{Participant interviews}

This interview was proposed to 38 subjects, but only 20 agreed to take part in it (6 men and 14 women). Only 3 (15\%)

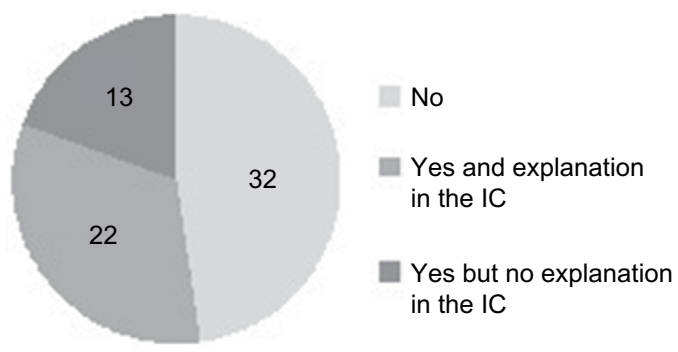

Figure I Provision of identification card to participants in the clinical trials. Abbreviation: IC, identification card.

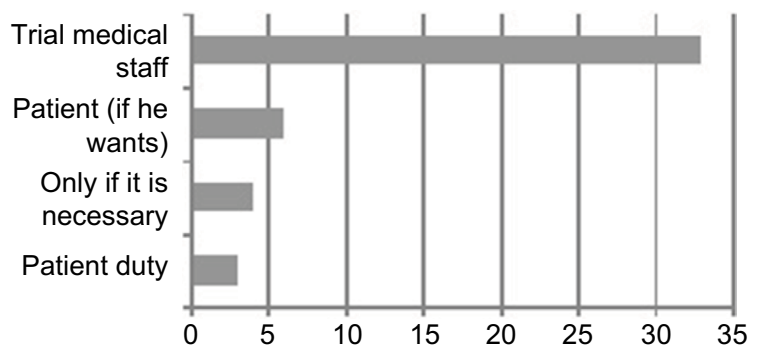

Figure 2 Preferred method to communicate to the family physician patient clinical trial participation.

knew the title of the study in which they were participating, $14(70 \%)$ were aware of their illness and $6(30 \%)$ could not answer either of these two questions. However, nearly all the participants (18/20) could remember the name of the PI.

Regarding the ID card (informing about the participation in a clinical trial), only $3 / 20(15 \%)$ participants declared that they had been provided with such identification at the beginning of the study. When the interviewed subjects were asked about their awareness of the convenience of informing their primary care physician with regard to their participation in the study, half of them responded negatively. However, the majority of subjects $(70 \%)$ pointed out that their primary care physician was aware of their inclusion in a clinical trial, mostly through personal communication with the PI through a letter or through a note in their electronic medical records. Nearly all the interviewed subjects (18/20) considered that their family physician should be informed of their inclusion in a clinical trial.

Finally, regarding the best way to inform primary care physicians, patients were asked to rate from 1 to 5,5 being the highest score, the different methods that could be used. The results of the scores for this part of the survey are showed in Figure 3. It must be noted that the option "Through electronic medical records" obtained the best score (93 of the maximum 100 points).

\section{Discussion}

Our results confirm that there is a significant number of clinical trials that do not correctly communicate the fact that the patients are being included in such a trial to the family physician. There is a scarcity of published work on this matter, although much attention has been devoted to the informed consent and how to use it. Some IRBs include an ID participation card as part of the documentation required for the clinical trial to be accepted for review. GPC guidelines state that "all subjects participating in a clinical trial with an Advanced Therapy Investigational Medicinal Product (ATIMP) should receive from the investigator an alert 


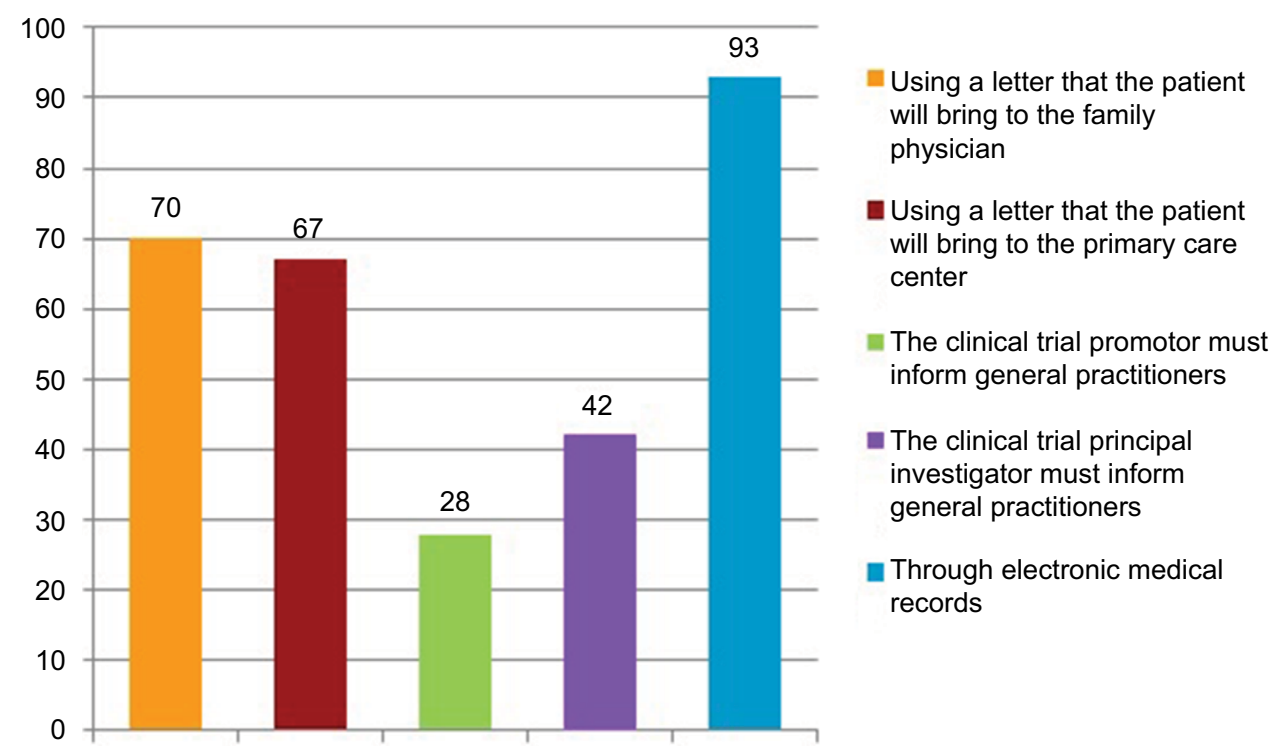

Figure 3 Participant's scores given to the different options available to communicate to the family physician and their inclusion in a clinical trial.

card, ... containing as minimum the name of the subject, the investigator contact number and information regarding the medical treatment received." 12

The lack of available basic information about the clinical trial characteristics, for instance the title and the PI contact information, can generate safety issues for patients. ${ }^{13,14}$ Most of the trials we have included in the survey recruit patients with a chronic illness (e.g., diabetes) or cancer. These patients could have acute exacerbations of their illness or experiment adverse effects that would need attention in emergency care facilities; without adequate information about what can be done and what interventions must be avoided, there is a risk of the attending medical staff making erroneous decisions, that at worst could result in fatalities and at best in the patient exclusion from the trial. In these cases, it is critical to have information about the adverse reactions that the experimental treatment can induce and how to manage them in order to avoid investing unnecessary efforts and valuable time into investigating the causes of the clinical manifestations that the patient presents with. The use of an ID card, which includes basic information about the trial, is the easiest and most effective way to provide such information. Although more than half of the protocols included in this study supplied participants with an ID card, only a minority of the interviewed participants declared that they had received such a card at the beginning of the study.

Most of the clinical trial participants who were interviewed did consider that their family physician should be informed about their participation in a trial. Due to their frequent contacts with the primary care staff, participants saw this as necessary. As a whole, nearly 2 out of 3 protocols did consider informing the general practitioner and, in most cases, the responsibility of doing so relied on the PI. Unfortunately, in the present study, we could not verify whether this action was really performed. It must be noted that in some protocols the responsibility of informing the family physician is transferred to the participant. In the case of our IRB, this is not accepted since it is considered that the best way to transfer the information is through direct communication between practitioners, either by using electronic medical records or personal letters. Moreover, our committee agrees not to inform the family doctor if the patient manifests explicitly against.

Our team has modeled these initiatives on those promoted by the clinical research nurses working for the Experimental Cancer Medicine Center Network; they care for patients participating in translational and early phase clinical trials across the Network. ${ }^{15}$ Some of the key ideas developed by this group of nurses are: 1) the wider use of patient wallet cards for clinical study ID and out-of-hours contact details, and 2) the use of patient electronic records to upload an alert notifying staff that the patient is taking part in a trial and to regularly update the research section.

As is acknowledged by most institutions, patients being admitted to a nontreating hospital - that is, not where the trial is being run - are still a barrier to best practice. Our center is working on a project to establish a formal procedure to inform general practitioners and emergency facilities about subjects' participation in a clinical trial. This procedure will warrant confidentiality for the subject and the clinical trial sponsor, and electronic health records will be the main tool. 
Nowadays, our health system's electronic records allow health information to be shared between specialized and primary care. The proposal is to create an alert that triggers when a patient is included in a clinical trial; this warning will contain basic information about the trial.

\section{Conclusion}

Information directed to health care practitioners who are not involved in clinical trials conducted by specialized medical staff is still scarce and there is great variability in the methods used to transmit such information. Although the provision of patient wallet cards for clinical study ID is widespread, the use of such identification remains questionable. Fortunately, there is a significant majority of clinical trial participants who recognize that their family physicians are aware of their inclusion in a clinical trial.

\section{Acknowledgments}

The authors would like to thank Elena Rodriguez-Murphy and Esther Rodriguez-Murphy for their translating the work and for style corrections. This work is to be attributed to the Institut de Recerca Biomèdica de Lleida (IRBLleida).

\section{Disclosure}

The authors report no conflicts of interest in this work.

\section{References}

1. Cox K, McGarry J. Why patients don't take part in cancer clinical trials: an overview of the literature. Eur J Cancer Care. 2003; 12(2):114-122.

2. Somkin CP,Altschuler A, Ackerson L, et al. Organizational barriers to physician participation in cancer clinical trials. Am JManag Care. 2005;11(7): $413-421$.
3. Comis RL, Miller JD, Colaizzi DD, et al. Physician-related factors involved in patient decisions to enroll onto cancer clinical trials. J Oncol Pract. 2009;5(2):50-56.

4. Long DG. Clinical trials. A family physician's perspective. Cancer. 1991;67(S6):1798-1799.

5. Dolor RJ, Schmit KM, Graham DG, et al. Guidance for researchers developing and conducting clinical trials in practice-based research networks (PBRNs). J Am Board Fam Med. 2014;27(6):750-758.

6. Taylor H. Public awareness of clinical trials increases: new survey suggests those conducting trials are doing a better job of informing potential participant of opportunities. Harris Interact Healthc News. 2004;4:1-9.

7. IOM (Institute of Medicine). A National Cancer Clinical Trials System for the 21st Century: Reinvigorating the NCI Cooperative Group Program. Washington, DC: The National Academies Press; 2010.

8. Association of Clinical Research Professionals (ACRP). CenterWatch 2008 national survey of study volunteer experiences. Paper presented at the ACRP 2009 Global Conference and Exhibition; April 24-28; 2009; Denver, CO.

9. Pretesting NIH clinical trial awareness messages: a focus study with patients, caregivers, and the general public. Bethesda, MD: National Institutes of Health; 2011.

10. Joshi VD, Oka GA, Kulkarni AA, et al. Public awareness and perception of clinical trials: quantitative study in Pune. Perspect Clin Res. 2013;4(3):169-174.

11. Schain WS. Barriers to clinical trials. Part II: knowledge and attitudes of potential participants. Cancer. 1994;74(Suppl 9):2666-2671.

12. European Commission. Enterprise and industry directorate-general. Detailed guidelines on good clinical practice specific to advanced therapy medicinal products. Consumer goods. ENTR/F/2/SF/dn D(2009) 35810. Brussels: Pharmaceuticals; 2009.

13. De Pablo López de Abechuco I, Gálvez-Múgica M, Aguilar-Jiménez M, et al. Compresión del consentimiento informado de ensayos clínicos: encuesta a pacientes y a voluntarios. Conference and abstracts book. ANCEI Congress; 2015:121-125; Zaragoza, Spain.

14. Solanilla M, Rumi L, Zapata A, et al. "Nivel de información recibido y comprendido en pacientes que participan en ensayos clínicos oncológicos". Conference and abstracts book. ANCEI Congress; 2014:99-100; Madrid, Spain

15. ECMC Research Nurse Network Group Steering Committee. Promoting patient safety in cancer clinical trials. UKONS Oncology Nursing Society Newsletter. Issue 28; 2014. Available from:http://ukons.org/ newsletters. Accessed March 15, 2017. 


\section{Supplementary material}

Age:

Sex:

Name of the trial/disease:

Clinical trial physician:

1. Related with your participation in the clinical trial, have you been given a clinical trial participation card?

Yes / No / Don’t know/refused

2. Has been reported in the trial consent form that your general practitioner will be informed?

Yes / No / Don’t know/refused

3. Does your general practitioner know that you are participating in a clinical trial?

Yes / No / Don't know/refused

4. If your answer is Yes, how did your general practitioner get the information?

5. Do you think that your general practitioner must know that you are participating in a clinical trial?

Yes / No

6. In your opinion, which is the best way to inform your general practitioner that you are involved in a clinical trial? (preference order: $5 \rightarrow$ first place, $1 \rightarrow$ last place)

That you or a relative deliver your general practitioner a letter received from the investigator.

That you or a relative send your primary health centre a letter provided from the investigator.

What would you prefer, an opened envelope or a closed one? (choose with a cross)

\section{Opened \\ Closed}

How would you prefer to deliver it, personally or by mail?

Personally

By mail

That the clinical trial investigator informs to the general practitioner.

That the sponsor informs to the general practitioner.

That the general practitioner could find the information in the electronic medical history projected as an alarm.

Figure SI Participation survey.

\section{Publish your work in this journal}

The Open Access Journal of Clinical Trials is an international, peerreviewed, open access journal publishing original research, reports, editorials, reviews and commentaries on all aspects of clinical trial design, management, legal, ethical and regulatory issues, case record form design, data collection, quality assurance and data auditing methodologies. The manuscript management system is completely online and includes a very quick and fair peer-review system, which is all easy to use. Visit http://www.dovepress.com/testimonials.php to read real quotes from published authors. 